Check for updates

Cite this: RSC Adv., 2018, 8, 23931

\title{
Simple, selective and fast detection of acrylamide based on glutathione $S$-transferase
}

\begin{abstract}
Madalina-Petruta Bucur, (D) Bogdan Bucur (iD * and Gabriel-Lucian Radu (D)
Acrylamide (AA) is a toxic compound formed in thermally prepared foods by Maillard reaction. Besides foods, AA may be found in cosmetic products as an impurity of the widely-used non-toxic polyacrylamide. We present a novel, fast and selective detection method based on the amperometric monitoring of the coupling reaction between reduced glutathione (GSH) and AA catalyzed by glutathione S-transferase (GST) to produce an electrochemically inactive compound. We have used electrodes modified with cobalt-phthalocyanine to monitor the decrease of GHS concentration at $+300 \mathrm{mV}$. Our system is simple, does not require supplementary substrates such as 1-chloro-2,4-dinitrobenzene (CDNB) nor have disadvantageous competitive kinetics characteristic to inhibition like signals. Using the optimum concentration of $100 \mu \mathrm{M} \mathrm{GSH}$ we have obtained a linear calibration graph from 7 to $50 \mu \mathrm{M}$ AA and a limit of detection of $5 \mu \mathrm{M} \mathrm{AA}$. The method is not affected by interfering compounds usually found in foods and was applied for real sample analysis.
\end{abstract}

Received 14th March 2018

Accepted 24th June 2018

DOI: $10.1039 / \mathrm{c} 8 \mathrm{ra02252f}$

rsc.li/rsc-advances

There is an important interest in food industry to monitor

\section{Introduction}

Acrylamide (AA) is a toxic and potential cancerogenic compound that is absorbed in the body where it forms adducts with haemoglobin, DNA or other macromolecules and is detoxified through reaction with glutathione (GSH) catalysed by glutathione-S-transferases (GST). ${ }^{1}$ AA is formed in foods by Maillard reaction between the amino group of the amino acid asparagine and carbonyl groups of glucose and fructose during frying/baking in low moisture conditions. ${ }^{2}$ The Panel on Contaminants in the Food Chain of the European Food Safety Authority (EFSA) has conducted a thorough review of AA risk in foods and found that the margins of exposure indicate a concern for neoplastic effects based on animal evidence, but the epidemiological associations have not demonstrated that AA is a human carcinogen. ${ }^{3}$ There are food products such as dry coffee substitutes, coffee, potato crisps, fried potatoes, toast bread with important variation depending on 'darkness level' and origin that have AA concentrations at ppm levels. ${ }^{3}$ Besides foods, AA is found in numerous other products as an impurity of the widely used, non-toxic polyacrylamide and thus the AA levels raise concerns and are regulated, e.g. AA maximum levels in cosmetics are set at $5 \mathrm{ppm}$ for the Australian market. ${ }^{4}$ Plastic surgery involving polyacrylamide based implants leads to serious health issues. ${ }^{5}$ AA leaking from various polyacrylamide products raises important environmental ${ }^{6}$ and agricultural ${ }^{7}$ issues and thus the AA must be analysed in a variety of samples besides foods.

National Institute of Research and Development for Biological Sciences, Centre of Bioanalysis, 296, Splaiul Independentei, 060031 Bucharest, Romania. E-mail: bucurica@yahoo.com; Fax: +(40)212200900; Tel: +(40)212200900 and reduce the AA concentrations in thermally prepared products using optimized preparation techniques. ${ }^{8}$ The performances of the analytical techniques based on chromatographic or electrophoretic separations coupled with mass spectrometry detectors are remarkable, ${ }^{9}$ but these techniques require expensive equipment and complicated sample pre-treatment steps that are not suitable for small and medium food producers. There are alternative analytical techniques such as enzyme-linked immunosorbent assay (ELISA) kits that are able to detect AA after sample clean-up using solid phase extraction (SPE) ${ }^{10}$ but covalent alkylation takes place during the immunoreaction of AA with its specific antibody mainly through the cysteine and histidine from the surface of the antibody. ${ }^{11}$ Biosensors based on detection mechanisms that mimic toxicological mechanism in vitro for detection of AA have been reported: $\mathrm{DNA}^{12}$ or haemoglobin ${ }^{13}$ form adducts with $\mathrm{AA}$ and change their electrochemical properties. However, the formed adducts are irreversible, which limits the biosensor design to disposable devices. Amidase could also be employed for development of biosensors based on the fact that this enzyme catalyses also the hydrolysis of AA among other substrates. ${ }^{14}$

Our proposed strategy for development of bioanalytical methods for AA detection is based on the use of the natural detoxification mechanism catalysed by GST (EC number 2.5.1.18): the reaction between the GSH and xenobiotic $\mathrm{AA}^{15}$ (Fig. 1A). Biosensors based on GST were previously developed for the analysis of anticancer drugs based on the competition between cisplatin and 1-chloro-2,4-dinitrobenzene (CDNB) for the same active site of the enzyme in their parallel reactions with $\mathrm{GSH},{ }^{16} \mathrm{CDNB}$ being a reagent used for spectrometric measurement of GST activity (Fig. 1B). The GST inhibition by 

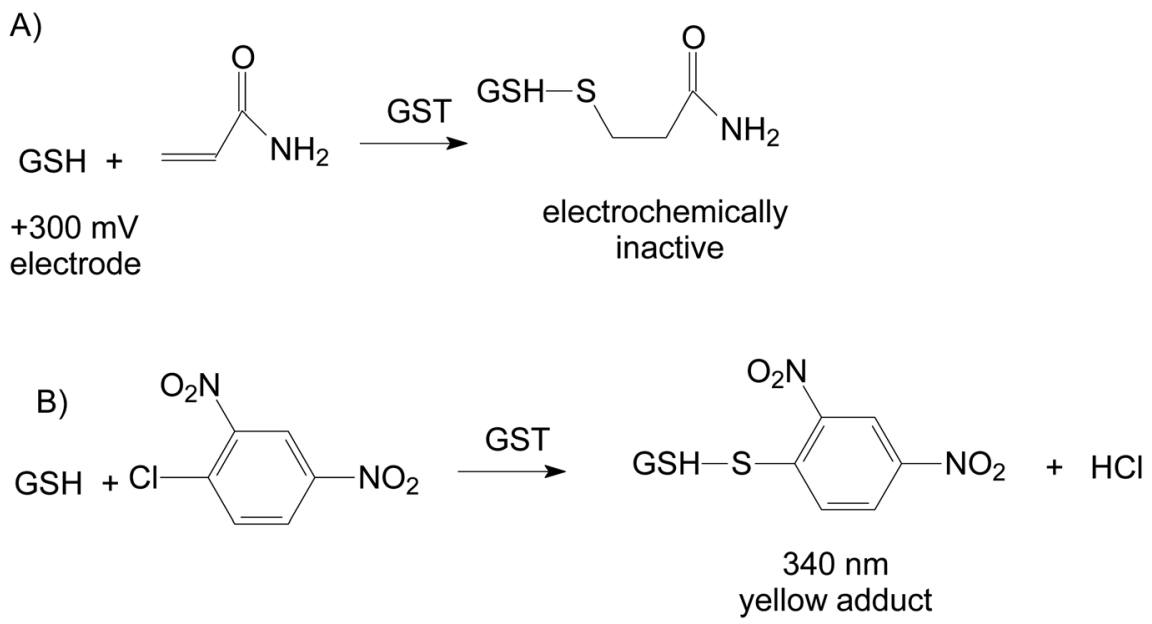

Fig. 1 The reactions catalysed by GST: (A) coupling of GSH with AA and (B) color reaction used for enzymatic activity measurements. A competition system may also be envisaged by the simultaneous reactions of both AA and CDNB with GSH leading to a decrease of the yellow product quantity in presence of AA.

insecticides was used for detection of malathion ${ }^{17}$ or molinate, ${ }^{18}$ compounds that should not be present in thermally prepared foods. To the best of our knowledge, this paper presents the first developed electrochemical enzymatic method for the analysis of AA from food samples based on analyte reaction with GSH catalysed by GST. We have used screen-printed electrodes modified with cobalt phthalocyanine for the amperometric quantification of GSH that reacts with AA to produce electrochemically inactive compounds. The decrease of the analytical signal depends on the concentration of AA and activity of GST.

\section{Materials and methods}

\subsection{Reagents}

Glutathione $S$-transferase (GST, EC number 2.5.1.18) from equine liver ( $25 \mathrm{UI} / \mathrm{mg}$ solid) was provided by Sigma-Aldrich (Germany), dissolved in phosphate buffer and stored at $-20{ }^{\circ} \mathrm{C}$ in the freezer for long term or at $+4{ }^{\circ} \mathrm{C}$ for a few days. Analytical grade cysteine, cysteamine, sodium phosphate dibasic, potassium phosphate monobasic, potassium chloride and starch, gluten, glucose, fructose, sucrose, sugar, citric acid, sodium benzoate and ammonium bicarbonate were purchased from Sigma-Aldrich. Standard stock solution of $50 \mathrm{mM}$ reduced glutathione (GSH), $100 \mathrm{mM}$ acrylamide (AA) and $100 \mathrm{mM} \mathrm{1-}$ chloro-2,4-dinitrobenzene (CDNB) all from Sigma-Aldrich, Germany were prepared daily in phosphate buffer. The phosphate buffer solution $0.05 \mathrm{M}$ (PBS) with $\mathrm{pH} 6.6$ (optimum value according to the enzyme producer) was supplemented with $\mathrm{KCl}$ $0.1 \mathrm{M}$ for electrochemical measurements.

\subsection{Apparatus}

The amperometric and cyclic voltammetry measurements were performed with a galvanostat/potentiostat Autolab PGSTAT302N (Metrohm-Autolab) controlled by a PC with the software Nova 1.8. The screen-printed electrodes were produced by BAE Laboratory, Université de Perpignan Via Domitia (France) on a plastic substrate and have a round working electrode with a diameter of $\sim 4 \mathrm{~mm}$ produced using a cobaltphthalocyanine modified paste as electrochemical mediator purchased from Gwent Electronic Materials (Gwent, UK), an auxiliary electrode made with carbon based ink and a $\mathrm{Ag} / \mathrm{AgCl}$ pseudo-reference electrode both produced with pastes from Acheson (Plymouth, UK).$^{19}$ The electromagnetic noise produced by magnetic stirring was reduced with the filter from the extreme low currents module (ECD) set to $1 \mathrm{~s}$.

\subsection{AA spectrometric analysis}

The AA spectrometric analysis was made at $340 \mathrm{~nm}$ in a quartz cuvette. The used concentrations were: $100 \mu \mathrm{M} \mathrm{GSH}, 100 \mu \mathrm{M}$ GSH and variable concentrations of AA. The reaction was started by injection of 0.25 IU of GST and the reaction was followed for $10 \mathrm{~min}$. The analytical signal is the variation of the absorbance per minute from the linear part and was correlated with the AA concentration. From the analytical signals were subtracted the blank values determined for measurements without enzyme.

\subsection{AA amperometric analysis}

The amperometric measurements of the enzymatic activity were made at $+300 \mathrm{mV}$ in $5 \mathrm{~mL}$ PBS under magnetic stirring. The current intensity was recorded and, after current stabilization, $100 \mu \mathrm{M}$ GSH (final concentration in the cell) was injected. The time necessary to reach the plateau was less than one minute. Various concentrations of AA from standard solutions or real samples were injected. The current intensity in the presence of GSH and AA without enzyme is the baseline signal. The reaction was started by the injection of 0.25 IU of GST. The measured current intensity decreases due to the reaction of AA with GSH in the presence of the enzyme. The analytical signal is the linear current decrease in time (nA $\mathrm{min}^{-1}$ ) and is correlated with AA concentration (or GSH/GST during optimization studies). From the analytical signals were subtracted the blank valued determined for measurements without enzyme. 


\subsection{Real sample analysis}

AA was determined in various food samples from a local store: bread, potato chips and homemade boiled potatoes. First, the food samples were crushed in a mortar and $5 \mathrm{~g}$ of food sample was mixed with $25 \mathrm{~mL}$ of ethanol for one hour. Then the ethanol was filtered and evaporated. The analytes were recovered in $10 \mathrm{~mL}$ in PBS and defatted with $2 \mathrm{~mL}$ of hexane to avoid electrode surface fouling. The real samples were analysed directly or spiked with known concentrations of AA (added before extraction).

\section{Results and discussions}

\subsection{Spectrometric determinations}

Preliminary tests were carried out using spectrometric measurements for the detection of AA. The activity of GST is measured spectrometrically at $340 \mathrm{~nm}$ using CDNB that reacts with GSH to produce a coloured compound: 2,4-dinitrophenyl$S$-glutathione (Fig. 1B). This system can be modified to include AA to be in competition with the chromogenic substrate CDNB for the enzymatic reaction with GSH: both reactions indicated in Fig. $1 \mathrm{~A}$ and $\mathrm{B}$ take place simultaneously. The measured absorbance is reduced in the presence of AA (inhibition-like signals). For known GSH and CDNB concentrations, the variation of the measured absorbance is dependent on the AA concentration from the sample. The decrease of absorbance can be considered an inhibition and a inhibition constant can be calculated. $^{20}$ This is based on the fact that the kinetics of the irreversible enzymatic reactions that have two or more substrates in competition can be mathematically described using the equations of the competitive inhibition kinetics by substituting the inhibition constants $K_{\mathrm{I}}$ with the respective ratio of the $K_{\mathrm{M}}$ values for each competing substrates ${ }^{21}$ as follows:

$$
V_{1}=\frac{V_{\max 1} \times S_{1}}{K_{\mathrm{M} 1}+\frac{K_{\mathrm{M} 1}}{K_{\mathrm{M} 2}} \times S_{2}+S_{1}}
$$

were 1 and 2 refers to the competing substrates AA and CDNB, respectively.

The spectrometric system based on CDNB is suitable for biological investigations of the AA toxicity and metabolism, ${ }^{20}$ but is not suitable for analytical purposes to reach low limits of detection. The limitation for analytical applications is due to the kinetics of the competitive reactions that result in very little competition when the concentrations of the two substrates are far below the $K_{\mathrm{M}}$, while the competition can be significant at substrate concentrations above $K_{\mathrm{M}} \cdot{ }^{21}$ The $K_{\mathrm{M}}$ values for both AA and CDNB are relatively high $(\sim \mathrm{mM})$ and in consequence the achievable limits of detection are unsatisfactory. This kinetic limitation is not encountered in the case of spectrometric measurements with specific enzymatic inhibitors such as dieldrin and spiromesifen insecticides ${ }^{22}$ or anticancer drugs such as cisplatin and oxoplatin that are well recognized by GST and detected by the competitive mechanism. ${ }^{\mathbf{1 6}}$ Our results have demonstrated that AA is not detectable at micromolar concentrations, significant signal decrease being obtained starting from $0.5 \mathrm{mM}$ AA. Thus, the spectrometric measurements are not adapted for AA analysis at low concentrations. In consequence, we have used electrochemical measurements of the GSH that do not imply a competition for the substrate.

\subsection{Electrochemical detection of GSH}

There are numerous electrodes modified with various electrochemical mediators reported for GSH detection. ${ }^{23}$ Prussian blue is a mediator suitable for food analysis ${ }^{24}$ that was reported to have a good sensitivity for the detection of $\mathrm{GSH} .{ }^{25}$ Thorough electrochemical studies have demonstrate that Prussian blue is an effective mediator for several thiolic compounds such as cysteamine or thiocholine, but ineffective for GSH. ${ }^{26}$ We have tested screen-printed electrodes modified with Prussian blue for GSH and found that the sensitivity was insufficient for detection at low concentrations (data not shown). In consequence, we have used screen-printed electrodes modified with cobalt-phthalocyanine for amperometric detection of GSH. We have compared the amperometric signals obtained for GSH with two other low mass natural thiolic compounds (cysteamine and cysteine) in order to investigate the electrocatalytic properties of the mediator towards thiols and asses the sensitivity differences that prevent the use of Prussian blue. The initial investigations were carried out by cyclic voltammetry and the screen-printed electrodes modified with cobalt-phthalocyanine were compared with similar carbon screen-printed electrodes without mediator. For the electrodes with cobalt-phthalocyanine we have obtained a large oxidation peak for cysteamine and cysteine with maximum $\sim 350 \mathrm{mV}$ and a wide but smaller peak for GSH with maxima starting at $300 \mathrm{mV}$ (Fig. 2). In order to demonstrate the effectiveness of the cobalt-phthalocyanine mediator, we have carried out blank test using electrodes made with a carbon paste without mediator and we have obtained only a very small oxidation peak at potentials higher than $650 \mathrm{mV}$ for cysteamine and cysteine (Fig. 2).

We have observed that the electrodes with cobalt-phthalocyanine allow the amperometric detection at $300 \mathrm{mV}$ of all three

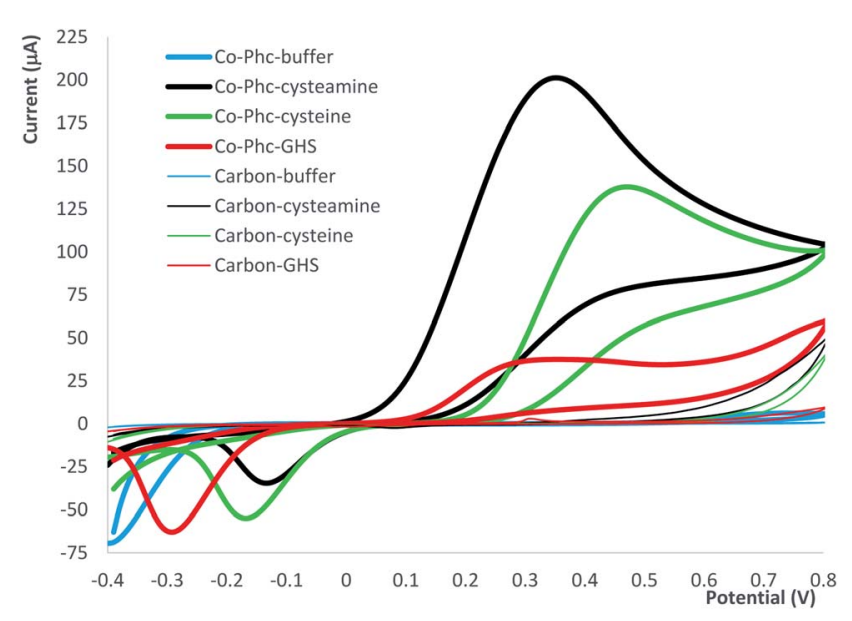

Fig. 2 Cyclic voltammograms obtained using electrodes modified with cobalt-phthalocyanine (Co-Phc) in comparison with carbon screen-printed electrodes (carbon). The experiments were carried out in buffer and in buffer solution with $10 \mathrm{mM}$ cysteamine, cysteine or GSH. 
investigated thiolic compounds and the measurement sensitivity decreases in the following order: cysteamine, cysteine and GSH (see equations of the calibration graphs in Fig. 3). The calibration graph for GSH was linear from 5 to $900 \mu \mathrm{M}$ with a measurement sensitivity of $\sim 3.8 \mathrm{nA} \mu \mathrm{M}^{-1} \mathrm{GSH}$ and a detection limit of $1 \mu \mathrm{M}$ calculated as $3 \times$ signal/noise. The reproducibility of the measurements was tested by injecting $100 \mu \mathrm{M}$ GSH and the recorded analytical signal was $316 \pm 11 \mathrm{nA}$, corresponding to a relative deviation standard $\mathrm{RSD}=3.5 \%(n=9)$. These analytical figures of merit allow an amperometric detection of GSH with satisfactory performances in terms of concentration range and magnitude of analytical signals for development of enzymatic detection of AA based on GST and electrochemical quantification of enzymatic substrate GSH using mediated amperometric measurements.

\subsection{Determination of AA using GST}

The main parameter to be optimized is the substrate concentration. The proposed amperometric detection of AA is based on the irreversible reaction between AA and GSH that is catalysed by GST. The enzymatic kinetic for a two-substrate reaction depends on the concentration of both substrates ${ }^{27}$ according to the eqn (2):

$$
V=\frac{V_{\max } \times S_{1} \times S_{2}}{K_{1} \times K_{2}+K_{2} \times S_{1}+K_{1} \times S_{2}+S_{1} \times S_{2}}
$$

where 1 and 2 refers to GSH and AA, respectively; $K_{1}, K_{2}$ are the equilibrium constants of the corresponding GST-substrate complexes. Eqn (2) becomes classic Michaelis-Menten if one substrate (i.e. GSH) is in constant or large excess concentration (eqn (3)):

$$
V_{2}=\frac{V_{\max }^{\prime} \times S_{2}}{K_{\mathrm{M}}^{\prime}+S_{2}}
$$

where the pseudoconstants $V_{\max }^{\prime}$ and $K_{\mathrm{M}}^{\prime}$ depend on GSH concentration.

In consequence, one important factor for the analytical performances is the concentration of GSH. A higher GSH

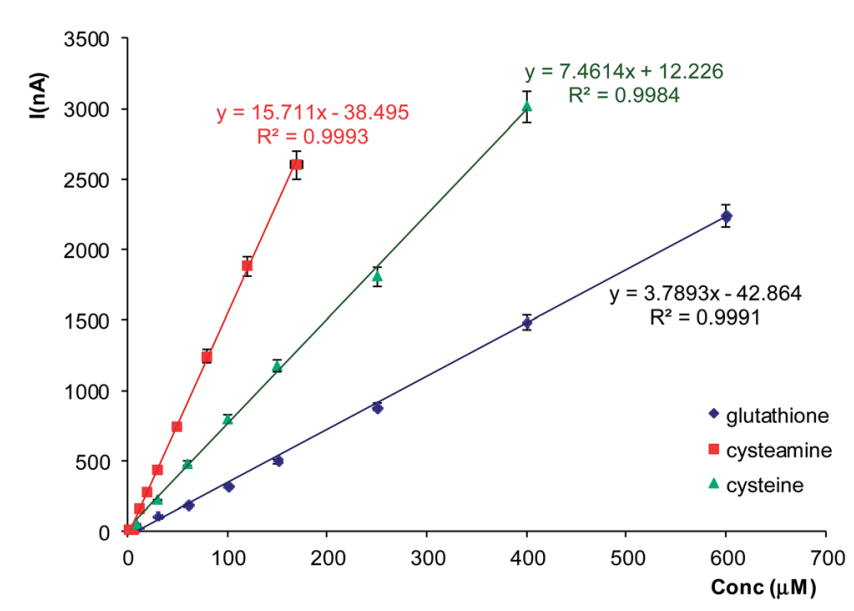

Fig. 3 The calibration curves obtained with screen-printed electrodes modified with cobalt-phthalocyanine at $300 \mathrm{mV}$ for cysteamine, cysteine and GSH. The experiments were carried out in triplicate. concentration implies an increase reaction rate with $\mathrm{AA}$, but also higher currents and an increased base-line. Moreover, GSH is expensive and an optimization of GSH concentration has to be done by taking into consideration also this aspect. We have tested three concentrations of GSH for AA detection: $50 \mu \mathrm{M}, 100$ $\mu \mathrm{M}$ and $200 \mu \mathrm{M}$. The calibration graph and limit of detection (LOD) were determined for AA for each of the tested GSH concentrations. The analytical signals (represented by the current reduction in time measured in $\mathrm{nA} \min ^{-1}$ ) have an increased magnitude for higher GSH concentrations (Fig. 4), but this is accompanied by an increase of the baseline (proportional with GSH concentration) and noise. For $50 \mu \mathrm{M}$ GSH we have obtained a linear calibration graph between 10 and $35 \mu \mathrm{M} \mathrm{AA}\left(\Delta I\left(\mathrm{nA} \min ^{-1}\right)=0.5215 \times \operatorname{conc}(\mu \mathrm{M})+3.2144 ; R^{2}\right.$ $=0.93 ; n=5$ ) with a LOD of $7 \mu \mathrm{M}$ AA (calculated as $3 \times$ signal $/$ noise). In the case of $100 \mu \mathrm{M}$ GSH we have achieved a wider calibration graph linear from 7 to $50 \mu \mathrm{M} \mathrm{AA}\left(\Delta I\left(\mathrm{nA} \mathrm{min}{ }^{-1}\right)=\right.$ $0.643 \times$ conc $\left.(\mu \mathrm{M})+9.9435 ; R^{2}=0.94 ; n=5\right)$ and a LOD of $5 \mu \mathrm{M}$ AA. For the highest tested concentration of $200 \mu \mathrm{M}$ we have obtained a calibration graph linear in the 10 to $50 \mu \mathrm{M}$ AA range $\left(\Delta I\left(\mathrm{nA} \min ^{-1}\right)=0.5968 \times \operatorname{conc}(\mu \mathrm{M})+25.663 ; R^{2}=0.92 ; n=5\right)$ with a corresponding LOD of $7 \mu \mathrm{M}$ AA. The values of the correlation coefficient $R^{2}$ are higher than 0.92 , were calculated for $n=5$ experimental points that were linear before the plateau (Fig. 4). The differences between the LOD and linear ranges of the calibration curves are caused by a combination of factors manly due to enzymatic kinetic and amperometric detection. Based on these results, we have chosen $100 \mu \mathrm{M}$ GSH as the optimum concentration for AA detection and used it for the rest of the paper. Similar optimization of the substrate concentration based on a compromise between the magnitude of the measured currents and the specific enzymatic kinetic conditions was previously reported for $\beta$-carbolines analysis using inhibition of monoaminoxidases, ${ }^{28}$ while in case of irreversible inhibition a high substrate concentration is used for maximum analytical signals. ${ }^{29}$ The reproducibility of the enzymatic measurements of AA using the optimum concentration of 100 $\mu \mathrm{M}$ GSH was tested for $25 \mu \mathrm{M}$ AA and the variation of the current was $28.6 \pm 2.1 \mathrm{nA} \min ^{-1}$, corresponding to a relative deviation standard RSD $=7.3 \%(n=7)$. The calibration graphs reported in literature for other biological analytical methods ranges from

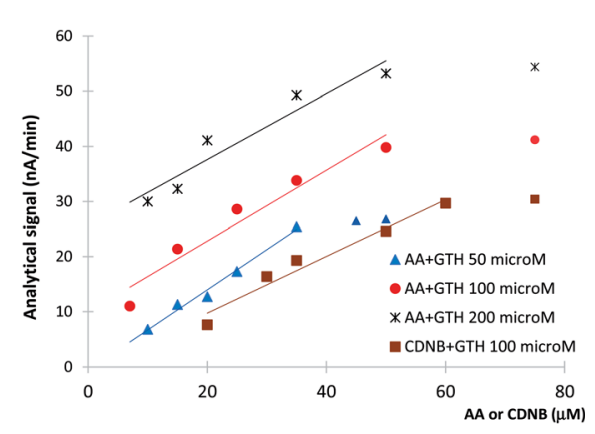

Fig. 4 The calibration curves for AA obtained with three different GSH concentrations. A comparison with CDNB is also presented using 100 $\mu \mathrm{M}$ GSH. The calibration curve is calculated using only the signals for the first 5 concentrations as a plateau is reached for higher concentrations. 
0.4-200 $\mu \mathrm{M}$ AA obtained by investigation of DNA-AA interactions, ${ }^{12} 0.04-10 \mu \mathrm{M}$ AA for electrodes based on haemoglobin ${ }^{13}$ to 0.14-14 $\mu \mathrm{M}$ AA for an immunological assay. ${ }^{10}$

We have carried out a comparison of AA detection with CDNB as a well-known substrate for spectrometric measurement of GST activity. Using the optimum concentration of 100 $\mu \mathrm{M}$ GSH we have obtained a calibration graph linear from 20 to $60 \mu \mathrm{M} \operatorname{CDNB}\left(\Delta I\left(\mathrm{nA} \min ^{-1}\right)=0.5148 \times \operatorname{conc}(\mu \mathrm{M})+0.5574 ; R^{2}\right.$ $=0.96$ ) and a LOD of $18 \mu \mathrm{M}$ CDNB (calculated as noise $/ 3 \times$ signal). These results demonstrate the validity of our proposed detection mechanism based on amperometric quantification of GSH, the system being able to quantify the usually used spectrometric substrate and confirming the possibility to detect any GST substrate. The amperometric detection has the advantage of being useful for analysis of coloured solutions were CDNB is not applicable.

\subsection{Interference study and real samples analysis}

Interferences can affect the amperometric detection or interact with the enzyme. Several potentially interfering substances were investigated based on their possible presence in foods: starch, gluten, glucose, fructose, sucrose, sugar, citric acid, sodium benzoate and ammonium bicarbonate. The initial study was carried out without GST to measure the electrochemical interferences. After the baseline stabilization, the interfering substances were injected at a $10 \mathrm{mM}$ final concentration in the cell and no significant current variation was registered. Subsequently, it was injected $100 \mu \mathrm{M}$ GSH and the recorded current was similar with the one obtained in the absence of the interferent. The substances that are known to interact with the enzyme (some anticancer drugs and the insecticides mentioned in the introduction) are not found in foods prepared by long thermal treatments.

Real food samples were analysed using the developed amperometric method based on GST. Thermally prepared foods were tested: bread and potato chips (from a local store as potentially contaminated products) and homemade boiled potato (as a blank sample because AA is usually formed by frying or baking). The samples were analysed directly or spiked with $1.5 \mu \mathrm{g} \mathrm{AA} / \mathrm{g}$ sample before the extraction steps. We did not detect AA in the real samples. The recovery percentages of the spiked AA were between 92 and 105\% (see Table 1).

Table 1 Real sample analysis using the proposed enzymatic method. Results are the average of 5 measurements

\begin{tabular}{lll}
\hline Sample & AA found $\left(\mu \mathrm{g} \mathrm{g}^{-1}\right)$ & Recovery $(\%)$ \\
\hline Bread & $<$ LOD & - \\
Bread spiked with & $1.42 \pm 0.12$ & 95 \\
$1.5 \mu \mathrm{g}$ AA/g & & \\
Potato chips & $<$ LOD & - \\
Potato chips spiked with & $1.38 \pm 0.14$ & 92 \\
$1.5 \mu \mathrm{g}$ AA/g & & \\
Boiled potato & $<$ LOD & - \\
Boiled potato spiked with & $1.57 \pm 0.15$ & 105 \\
$1.5 \mu \mathrm{g}$ AA/g & &
\end{tabular}

\section{Conclusions}

We have developed a simple and effective enzymatic method for AA based on GST and a direct coupling enzymatic reaction that mimic the natural detoxification mechanism. Interestingly, our system is simple and does not require the presence of both CDNB and GSH to measure inhibition like signals as other reported GSH based electrochemical biosensors for detection of molinate $^{\mathbf{1 8}}$ or anticancer drugs ${ }^{\mathbf{1 6}}$ and therefore benefit from an optimized enzymatic kinetics as detailed above. The choice of different enzymes extracted from various sources organisms with higher catalytic activity or even genetically engineered variants ${ }^{17}$ should allow a further improvement of the analytical figures of merit. The proposed enzymatic method can be used for the screening of AA in thermally prepared food samples or cosmetics with high AA content, ${ }^{4}$ is simple, easy to use and can be implemented in medium equipment laboratory (only a potentiostat and a freezer for reagent storage is required).

\section{Conflicts of interest}

There are no conflicts to declare.

\section{Acknowledgements}

We are grateful to prof. Jean Louis Marty from BAE Laboratory, Université de Perpignan Via Domitia (France) for the screenprinted electrodes with cobalt-phthalocyanine mediator. Paper revision by Dr Alina Vasilescu is gratefully acknowledged. This work was financed by the program Partnership in priority areas - PN II, supported by the Romanian Department of Education and Research (MEN-UEFISCDI) projects no. PN-IIIP2-2.1-PED-2016-0367 and PN-III-P2-2.1-PED-2016-0503.

\section{References}

1 N. Duale, T. Bjellaas, J. Alexander, G. Becher, M. Haugen, J. E. Paulsen, H. Frandsen, P. T. Olesen and G. Brunborg, Toxicol. Sci., 2009, 108, 90-99.

2 Y. Yuan, Z. Huanjie, D. Jie, M. Yutian, C. Fang and H. Xiaosong, RSC Adv., 2014, 4, 6608.

3 EFSA Panel on Contaminants in the Food Chain (CONTAM), EFSA J., 2015, 13, 4104.

4 Cosmetic Ingredient Review Expert Panel, Int. J. Toxicol., 2005, 24(suppl. 2), 21-50.

5 A. Manafi, A.-H. Emami, A. H. Pooli, M. Habibi and L. Saidian, Aesthetic Plast. Surg., 2010, 34, 413-422.

6 A. G. Guezennec, C. Michel, K. Bru, S. Touze, N. Desroche, I. Mnif and M. Motelica-Heino, Environ. Sci. Pollut. Res., 2015, 22, 6390-6406.

7 E. Mroczek, T. Kleiber, P. Konieczny and A. Waśkiewicz, Food Addit. Contam., Part A, 2015, 1-7.

8 Y. Yuan, Z. Huanjie, M. Yutian and Z. Hong, RSC Adv., 2014, 4, 1004-1009.

9 I. Pugajeva, J. Jaunbergs and V. Bartkevics, Food Addit. Contam., Part A, 2015, 32, 170-179. 
10 M. Franek, D. Rubio, I. Diblikova and F. Rubio, Talanta, 2014, 123, 146-150.

11 S. Zhou, D. Wang, C. Zhang, Y. Zhao, M. Zhao and Y. Wu, J. Immunoassay Immunochem., 2015, 36, 295-311.

12 S. Huang, S. Lu, C. Huang, J. Sheng, L. Zhang, W. Su and Q. Xiao, Sens. Actuators, B, 2016, 224, 22-30.

13 S. Garabagiu and G. Mihailescu, J. Electroanal. Chem., 2011, 659, 196-200.

14 S. Martins, A. Karmali and M. L. Serralheiro, Anal. Biochem., 2006, 355, 232-239.

15 R. N. Armstrong, Chem. Res. Toxicol., 1991, 4, 131-140.

16 E. M. Materon, P.-J. Jimmy Huang, A. Wong, A. A. Pupim Ferreira, M. D. P. T. Sotomayor and J. Liu, Biosens. Bioelectron., 2014, 58, 232-236.

17 P. Kapoli, I. A. Axarli, D. Platis, M. Fragoulaki, M. Paine, J. Hemingway, J. Vontas and N. E. Labrou, Biosens. Bioelectron., 2008, 24, 498-503.

18 T. I. S. Oliveira, M. Oliveira, S. Viswanathan, M. Fátima Barroso, L. Barreiros, O. C. Nunes, J. A. Rodrigues, P. de Lima-Neto, S. E. Mazzetto, S. Morais and C. Delerue-Matos, Talanta, 2013, 106, 249-254.
19 Z.-H. Li, H. Guedri, B. Viguier, S.-G. Sun and J.-L. Marty, Sensors, 2013, 13, 5028-5039.

20 R. Dixit, H. Mukhtar, P. K. Seth and C. R. Krishna Murti, Biochem. Pharmacol., 1981, 30, 1739-1744.

21 S. Schäuble, A. K. Stavrum, P. Puntervoll, S. Schuster and I. Heiland, FEBS Lett., 2013, 587, 2818-2824.

22 E. G. Chronopoulou, A. C. Papageorgiou, A. Markoglou and N. E. Labrou, J. Mol. Catal. B: Enzym., 2012, 81, 43-51.

23 J. C. Harfield, C. Batchelor-McAuley and R. G. Compton, Analyst, 2012, 137, 2285.

24 M.-P. Bucur, M.-C. Radulescu, B. Bucur and G. L. Radu, Anal. Sci., 2016, 32, 135-140.

25 A. Fagan-Murphy, M. C. Allen and B. A. Patel, Electrochim. Acta, 2015, 152, 249-254.

26 F. Ricci, F. Arduini, A. Amine, D. Moscone and G. Palleschi, J. Electroanal. Chem., 2004, 563, 229-237.

27 R. L. Stein, Kinetics of Enzyme Action, John Wiley \& Sons, Inc., Hoboken, NJ, USA, 2011, pp. 141-168.

28 M.-C. Radulescu, M.-P. Bucur, B. Bucur and G. L. Radu, Talanta, 2015, 137, 94-99.

29 J.-S. Do and K.-H. Lin, J. Taiwan Inst. Chem. Eng., 2016, 63, 25-32. 